\title{
Perfil da Amamentação em Lactantes Atendidas na Rede Básica de Saúde do Município de Ji-Paraná - RO
}

\author{
Isabele Ferreira Lisboa ${ }^{1}$, Jaqueliny dos Reis Vaz ${ }^{2}$, Francieli Carniel ${ }^{3}$
}

\begin{abstract}
RESUMO
Este é um estudo transversal, exploratório e com abordagem quantitativa, com objetivo de traçar o perfil da amamentação em lactantes atendidas na rede básica de saúde de Ji-Paraná - RO. A amostra foi composta por cem lactantes que frequentaram as unidades básicas de saúde (UBS). Os dados foram coletados por meio de um questionário composto e adaptado de Nogueira (2009), aplicado no período de julho a agosto de 2016. Os resultados obtidos mostraram que as lactantes tinham entre 20 e 30 anos, 90\% viviam com seu companheiro, 38\% possuíam Ensino Médio completo e apenas 7\% o Ensino Superior completo, $61 \%$ sem vínculo empregatício, $54 \%$ era a primeira gestação, 52\% possuíam dificuldades para amamentar, $95 \%$ acreditavam que o aleitamento materno exclusivo e o colostro são importantes e $100 \%$ realizaram o pré-natal na última gestação. Em relação a quem forneceu informações sobre amamentação para as lactantes a predominância foi de enfermeiros (70\%). Conclui-se que as mães possuem conhecimento sobre amamentação. Mesmo a sua maioria sendo jovens e na primeira gestação e apresentando algumas dificuldades, elas sempre estão dispostas a dar o seu melhor para o filho.
\end{abstract}

Palavras-chave: Amamentação. Lactante. Aleitamento materno.

\section{PROFILE OF THE BREASTFEEDING IN NURSING MOTHERS ATTENDED BY THE BASIC HEALTH SERVICE OF JI-PARANÁ - RO}

\section{ABSTRACT}

This is a transversal, exploratory and with quantitative approach study, which the objective is outline the profile of the breastfeeding in nursing mothers attended by the basic health service of Ji-Paraná - RO. The sample was composed by 100 nursing mothers who went to the Basic Health Unit. The data was collected through a survey composed and adapted of Nogueira (2009), applied in the period from July to August of 2016. The results obtained showed that the nursing mothers had between 20 and 30 years old, $90 \%$ lived with their partners, $30 \%$ completed high school and only $7 \%$ completed the university education, $61 \%$ do not have employment relationship, 54\% are first pregnancy, 52\% have difficulties to nurse, $95 \%$ believed that exclusive breastfeeding and colostrum are important, and $100 \%$ completed the prenatal on the last pregnancy. In relation to who gave the information about breastfeeding to the nursing mothers, the predominance was of nurses $(70 \%)$. The conclusion is that mothers have knowledge about breastfeeding, even with the majority being young and first pregnancy and presenting some difficulties, they are always inclined to give the best for their children.

Keywords: Breast-feeding. Lactating. Breastfeeding.

Recebido em: 30/8/2017

Revisões requeridas em: $16 / 5 / 2018$

Aceito em: 7/8/2018

\footnotetext{
${ }^{1}$ Enfermeira Pós-Graduanda em Auditoria de Enfermagem. izasf-29@hotmail.com

2 Enfermeira Pós-Graduanda em Obstetrícia e Ginecologia. jaqueliny.vaz@hotmail.com

${ }^{3}$ Orientadora. Enfermeira formada pela UFPel. Docente do curso de Enfermagem do Ceulji/Ulbra. Especialista em Neonatologia pela PUC/RS e em Pediatria pela UFRGS. Mestre em Genética e Toxicologia Aplicada. franci.carniel@gmail.com
} 


\section{INTRODUÇÃO}

Amamentar é um ato natural, que envolve vínculo, afeto, carinho, proteção e nutrição para o bebê. É um processo que desenvolve grande interação da mãe com o filho, repercutindo em seu desenvolvimento fisiológico, emocional, cognitivo e nutritivo, trazendo benefícios para a saúde da mãe e da criança, contribuindo, também, para a redução da mortalidade infantil (DIAS et al., 2015).

Segundo a Organização Mundial de Saúde (OMS), o aleitamento materno exclusivo (AME) é uma das ações básicas de saúde no combate à desnutrição e melhoria das condições de vida da população infantil. O AME deve ser mantido nos seis primeiros meses de vida, com o objetivo de suprir necessidades nutricionais (BRASIL, 2009).

$\mathrm{O}$ lactente deve receber o aleitamento materno (AM) com a complementação adequada de outros alimentos até os dois anos de idade ou mais, uma vez que é um mecanismo capaz de assegurar crescimento e desenvolvimento adequados, combate à mortalidade infantil por enfermidades comuns da infância e ser um alimento econômico (SOUZA; MENDES; BINOTI, 2016).

Estudos indicam que, no Brasil, a duração do AME é curta, a prevalência é baixa e o aleitamento materno exclusivo até os seis meses é raro. Hábitos inadequados, como o uso de chupetas e mamadeiras e a introdução precoce da alimentação complementar $(A C)$, incentivam o desmame precoce, trazendo inúmeros prejuízos para a saúde das crianças (SOUZA; MENDES; BINOTI, 2016).

Na AME nos primeiros seis meses de vida faz-se necessário informar e conscientizar a população geral quanto ao valor desta prática, melhorar os processos de trabalho dos serviços de saúde, dar suporte às mães na comunidade e em seus locais de trabalho e restringir a promoção inadequada de fórmulas lácteas infantis (CARVALHO; GOMES, 2017).

Visando a que as informações e o suporte dado às mães, de forma individual ou em grupo, têm-se mostrado significativamente eficazes na promoção do AME, é de grande importância a capacitação de profissionais de saúde, agentes comunitários de saúde e outras pessoas que se propõem a dar suporte às mães que estão amamentando (CARVALHO; GOMES, 2017).

As adolescentes e adultas primigestas estão propícias a realizar práticas inadequadas com seu fiIho. Por estarem em sua primeira gestação, não terem experiência e não saberem como reagir a algumas situações, as mesmas se encontram vulneráveis a ade- rir a métodos de cuidados e de alimentações incorretos para seu bebê, como automedicação e introdução de outros alimentos na dieta da criança (MELO; SCHERMANN, 2012).

$\mathrm{O}$ ato de amamentar envolve na puérpera aspectos psicológicos, econômicos, culturais e biológicos. Estes, somados, podem intervir ou não na prática do aleitamento materno. Por isso, destaca-se a importância de realizar ações que ajudem a mãe a identificar e enfrentar os fatores que podem dificultar a amamentação (DIAS et al., 2016).

Com a prática do aleitamento materno a puérpera poderá perceber várias vantagens, como a diminuição de lóquios no pós-parto, rápida involução uterina, recuperação do peso em menos tempo, menor risco de câncer de ovário e de mama e o retardo no aparecimento da ovulação em razão da amenorreia lactacional (DIAS et al., 2016).

Segundo a OMS, estima-se que o aleitamento materno poderia evitar $13 \%$ das mortes em crianças menores de cinco anos em todo mundo por causas preveníeis. $O$ aleitamento materno traz benefícios principalmente para o bebê, evitando doenças como diarreia e infecção respiratória, diminui o risco de alergias e de diabetes, reduz a chance de obesidade, melhora a nutrição, melhora o desenvolvimento da cavidade bucal e ainda há evidências de que o aleitamento materno contribui para o desenvolvimento cognitivo (BRASIL, 2009). Com isso se faz o seguinte questionamento: Qual o perfil das lactantes atendidas na rede básica de um município do Norte do país?

Diante do exposto, o presente estudo tem por objetivo traçar o perfil das nutrizes atendidas na rede básica de saúde em uma cidade no Norte do Brasil e características relacionadas ao aleitamento materno. Os conhecimentos destes dados podem ser úteis para auxiliar na melhoria da qualidade do atendimento às puérperas e incentivar a promoção da prática de amamentação, contribuindo para a qualidade de vida materna e infantil.

\section{METODOLOGIA}

Trata-se de uma pesquisa transversal, exploratória e com abordagem quantitativa, realizada em cinco unidades básicas de saúde (UBS) em um município do Norte do país.

A coleta de dados ocorreu no período de juIho a agosto de 2016, no momento em que as nutrizes buscavam atendimento na UBS para consulta de puericultura, vacinação e/ou teste do pezinho nos lactentes de até seis meses de idade. Foram excluídas as 
mulheres que não aceitaram responder o instrumento de pesquisa, não apresentaram condições físicas e/ou psíquicas para realizar a entrevista e aquelas que não aceitaram assinar o Termo de Consentimento Livre e Esclarecido (TCLE), totalizando, ao final, uma amostra de cem mães.

O instrumento de pesquisa foi adaptado de Nogueira (2009) e era composto por 69 questões, abertas e fechadas, relacionadas à mãe e à amamentação. As questões referentes à mãe envolviam características socioeconômicas e dados obstétricos. As perguntas sobre amamentação continham informações como: orientações sobre AM, se a mãe amamentou o bebe, tempo de aleitamento materno exclusivo, fatores que dificultaram ou impediram de amamentar, onde receberam orientações sobre amamentação e dúvidas a respeito do tema.

A entrevista somente foi efetivada após o aceite e assinatura do TCLE, em atendimento à resolução 466/12 do Conselho Nacional de Saúde. O projeto foi submetido à apreciação do Comitê de Ética em Pesquisa com seres humanos (CEP) do Ceulji/Ulbra, com protocolo de aprovação 1.655.066.

Após revisão e codificação dos dados, os mesmos foram duplamente digitados e, posteriormente, comparados por meio do programa do Microsoft Excel $^{\circledast}$ 2013, para evitar perdas e/ou duplicidade dos dados. A análise dos dados envolveu procedimentos de estatística descritiva que foram expressos por meio de tabelas.

\section{RESULTADOS E DISCUSSÃO}

Em relação às características sociodemográficos das mulheres lactantes pesquisadas, observou-se que a média de idade foi 26,37 anos, sendo a maior idade 46 e a menor 15 anos. Os achados revelaram o predomínio de mulheres jovens, sendo a faixa etária com maior incidência entre 20 e 30 anos, com 61\% das mães, sendo compatíveis com a pesquisa realizada por Teter, Oselam e Neves (2015), na cidade de Curitiba, na qual verificou que a maioria, $55 \%$ das lactantes entrevistadas, era da faixa etária entre 19 a 30 anos. Já nos estudos de Silva (2013), as entrevistadas se encontravam na faixa etária entre 20 a 34 anos. Em contraponto, Dias et al. (2015) verificaram, em sua pesquisa, uma população mais velha, com $30,30 \%$ das mães entrevistadas na faixa etária entre 29 a 36 anos de idade.

Santos (2012) observou que na prática da amamentação as mães jovens mostraram-se comprometidas e dispostas a superar os desafios encontrados e, dentro do seu contexto de vida, pretendem desempenhá-la corretamente. Neste estudo, a maioria das lactantes entrevistadas (52\%) queixaram-se de dificuldades durante a amamentação, como ingurgitamento mamário, mastite, algia mamária, mamilo plano e invertido e hipogaláctia, o que nos leva a acreditar que esses fatores podem dificultar a permanência da amamentação.

Melo e Schermann (2012), em contrapartida dos estudos de Santos (2012), afirmam que as dificuldades apresentadas em relação ao cuidado do bebê se correlacionam com a falta de experiência das mães adolescentes. Por isso, destaca-se a grande importância das orientações do enfermeiro a respeito de como cuidar do bebê, como segurá-lo, técnicas de amamentação, identificar os choros que significam sede, fome, frio, calor, dor e outros.

\section{Tabela 1 - Perfil Sociodemográfico das Nutrizes de Ji-Paraná - RO}

\begin{tabular}{lcc} 
& FA & FR \\
Id ade & 15 & $15 \%$ \\
$15-19$ anos & 61 & $61 \%$ \\
$20-30$ anos & 21 & $21 \%$ \\
$31-40$ anos & 3 & $3 \%$ \\
Mais que 40 anos & & \\
$* 26,37$ & & \\
$* * 46$ & & \\
$* * * 15$ & & \\
\hline Estado Civil & 90 & $90 \%$ \\
\hline Vive com o companheiro & & $5 \%$ \\
Tem companheiro, mas não & 5 & $5 \%$ \\
vive com ele. & 5 & \\
Näo tem companheiro & & $22 \%$ \\
Escolaridade & 22 & $2 \%$ \\
\hline Fundamental Incomple to & 2 & $38 \%$ \\
Fundamental Completo & 38 & $18 \%$ \\
Médio Completo & 18 & $17 \%$ \\
Medio Incompleto & 17 & $7 \%$ \\
Superior Incompleto & 7 & \\
Superior Completo & & $39 \%$ \\
Trabalho remunerado & 39 & $61 \%$ \\
\hline Sim & 61 & \\
Näo & & \\
& &
\end{tabular}

FA: Frequência absoluta; FR: Frequência relativa; ${ }^{*}$ média de idade; ${ }^{* *}$ maior idade; ${ }^{* * *}$ menor idade

Fonte: Dados da pesquisa, 2016.

Ao serem questionadas sobre o estado civil, $90 \%$ das lactantes entrevistadas alegam viver com seus companheiros, o que é semelhantes aos dados encontrados no estudo de Dias et al. (2015), no qual $54,55 \%$ das lactantes eram casadas e $39,39 \%$ viviam em união estável, considerando um total de $93 \%$ de mulheres que vivem com seu companheiro.

Segundo Primo e Leite (2015), os companheiros das puérperas são considerados indispensáveis para o incentivo à amamentação. As lactantes relatam que 
com a participação dos companheiros a amamentação passa ser mais prazerosa, principalmente ao sentarem do seu lado nesse momento. Ressaltam, ainda, que, uma vez iniciado o aleitamento materno, é importante que os pais estabeleçam o apoio verbal e elogios à puérpera assegurando uma amamentação positiva.

Considerando o grau de escolaridade das mães, os dados encontrados neste estudo evidenciam uma incidência de que $38 \%$ das lactantes possuem o Ensino Médio completo. Esse resultado corrobora o estudo de Teter, Oselam e Neves (2015), em que somente 7\% das lactantes possuem Ensino Superior completo, o que se sugere que quanto maior o grau de escolaridade da mãe, maior a duração do aleitamento materno.

Molina, Gil e Victoriano (2013) afirmam que a baixa escolaridade é um dos principais fatores que levam ao desmame precoce, pois, quanto maior a escolaridade, maior o entendimento da importância e dos benefícios da amamentação. O nível de escolaridade das mães está relacionado à promoção do aleitamento materno e ao retardo da introdução precoce de outros alimentos na criança (REIS; GONÇALVES, 2016).

Um estudo de Coimbra e Rieth (2016) revelou que mães com maior tempo de estudo demonstram ter maior conhecimento sobre aleitamento materno. A baixa escolaridade está interligada à interrupção da amamentação exclusiva. Isto possivelmente ocorre porque mães com maior nível de escolaridade têm mais acesso à informações e vantagens e mais autoconfiança para manter esta prática nos primeiros seis meses de vida do filho.

No que diz respeito à ocupação das mães entrevistadas, os dados mostram uma incidência de $61 \%$ de lactantes que não trabalham fora de casa. Resultado semelhante foi observado no estudo de Souza, Mendes e Binoti (2016), que constatou que 58,2\% das mães eram donas de casa. De acordo com os autores, as mulheres atualmente ocupam um espaço amplo na sociedade e possuem uma vida mais ativa que no passado, portanto o período de aleitamento materno acaba sendo curto em razão do menor tempo disponível para a prática de amamentação, predominando o maior tempo de aleitamento materno às lactantes que não trabalham fora de casa.

Infere-se que o fato de as mães pesquisadas não trabalharem fora e, assim, estarem sempre juntas ao seu bebe, propicie o aleitamento materno exclusivo, trazendo maior benefício tanto para o lactente quanto para a lactante. Isto contribui para a maior adesão à amamentação, tendo em vista que a melhor maneira de aprender a amamentar corretamente é a sua realização.
Quando questionadas sobre os antecedentes obstétricos e informações sobre a amamentação, obtivemos os dados apresentados na Tabela 2.

Tabela 2 - Antecedentes obstétricos e informações sobre o aleitamento

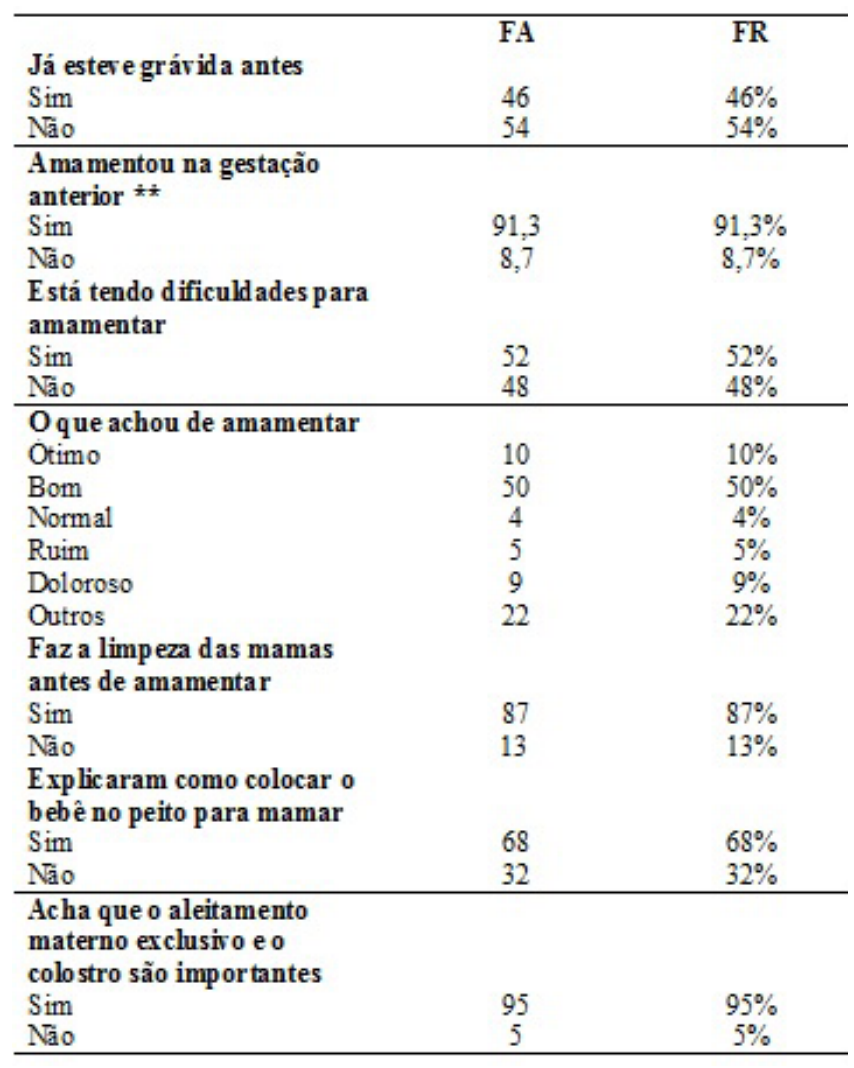

FA: Frequência absoluta; FR: Frequência relativa; **Cálculo referente direcionado somente às multíparas.

Fonte: Dados da pesquisa, 2016.

Quanto à paridade, os dados evidenciam uma discreta predominância de primíparas, com 54\% das entrevistadas. Segundo Oliveira e Oliveira (2012), as primíparas habitualmente têm mais dificuldades em relação à amamentação e cuidados com o lactente. A inexperiência de cuidar de um bebê pode justificar o pouco conhecimento dessas mulheres.

Relacionado à opinião sobre amamentar e se houve dificuldade no desempenho deste ato, cerca de $50 \%$ das mulheres questionadas responderam ser uma boa experiência, porém $52 \%$ responderam que, mesmo sendo bom, ainda é um momento difícil. Um estudo de Rocci e Fernandes (2014) mostra que as mães que apresentam complicações para amamentar desde a sua alta da maternidade obtiveram um porcentual de desmame precoce maior que as mães que não relataram problema algum, demonstrando, assim, que as dificuldades no processo de aleitamento estão fortemente associadas ao desmame precoce. 
As dificuldades no processo de amamentar quase sempre estão relacionadas aos cuidados inadequados com as mamas no período gestacional e puerperal. Esses podem causar complicações, contribuindo para o desmame precoce. Outro ponto que pode contribuir para o insucesso do aleitamento materno seria a posição e pega incorretas, pois o posicionamento e a pega adequada são de suma importância para que não ocorra o desmame precoce (SILVA et al., 2012).

Referente ao conhecimento da técnica correta de amamentação, $68 \%$ das entrevistadas lembram ter sido orientadas, e também sobre o preparo do seio antes de amamentar, e $87 \%$ afirmam realizar a limpeza das mamas antes da amamentação, resultado este que se contrapõe com um estudo realizado em 2015 na cidade de São Mateus-ES, que afirma que a maioria das mulheres desconhecem essas técnicas. As orientações sobre as devidas técnicas devem ser passadas às gestantes de forma individual ou coletiva, transmitindo, assim, informações e explorando o seu conhecimento prévio (VISITIN et al., 2015).

A técnica correta para amamentar é fundamental para um aleitamento materno confortável, pois, quando a criança pega a mama adequadamente, há uma abertura ampla da boca, abocanhando não apenas o mamilo, mas também a parte da aréola formando uma pega perfeita, garantindo a formação de um vácuo que é indispensável para que o mamilo e a aréola se mantenham dentro da boca do bebê. O posicionamento inadequado da boca do bebê interfere na dinâmica de sucção e extração do leite, o que poderá dificultar o esvaziamento da mama e gerar lesões mamilares, causando dor e desconforto para a mãe. Em razão desses problemas, é recomendável a orientação à mãe quanto à técnica de amamentação, realizando-a desde o pré-natal ou logo após o parto (CARVALHO; GOMES, 2017).

Apesar de aplicar corretamente toda a técnica de amamentação, alguns problemas podem surgir com a mama puerperal e é necessário que os profissionais de saúde os detectem o quanto antes a fim de resolvê-los, evitando que a mãe, por não conseguir superá-lo sozinha, desista de amamentar (CARVALHO; GOMES, 2017).

Sobre a importância do aleitamento materno exclusivo e colostro, observou-se, neste estudo, uma incidência de $95 \%$ das puérperas que acreditam na importância destes para o lactente. Segundo Visitin et al. (2015), o aleitamento materno exclusivo é um dos fatores principais para a saúde do bebê, fornecendo nutrição, imunidade e desenvolvimento da flora intestinal, considerada mais saudável. O colostro, que surge nos primeiros dias, contém todos os nutrientes que o lactente precisa, seguido pelo leite maduro que deve ser exclusivo até o sexto mês de vida, sendo desnecessária a suplementação alimentar até essa idade.

Tabela 3 - Informações sobre parto e pós-parto das lactantes

\begin{tabular}{|c|c|c|}
\hline $\begin{array}{l}\text { Fez pré-natal desta gestação } \\
\text { Sim } \\
\text { Näo } \\
\text { Fez pré-natal em qual } \\
\text { unidade de saúde }\end{array}$ & $\begin{array}{c}100 \\
0\end{array}$ & $\begin{array}{c}100 \% \\
0 \%\end{array}$ \\
\hline $\begin{array}{l}\text { UBS } \\
\text { Particular } \\
\text { Quem te passou informações } \\
\text { durante as consultas do pré- } \\
\text { natal }\end{array}$ & $\begin{array}{l}84 \\
16\end{array}$ & $\begin{array}{l}84 \% \\
16 \%\end{array}$ \\
\hline $\begin{array}{l}\text { Medico } \\
\text { Enfermeiro } \\
\text { Outros }\end{array}$ & $\begin{array}{c}22 \\
70 \\
8 \\
\end{array}$ & $\begin{array}{l}22 \% \\
70 \% \\
8 \% \\
\end{array}$ \\
\hline $\begin{array}{l}\text { Existem vantagens para a } \\
\text { mulher que amamentar }\end{array}$ & & \\
\hline $\begin{array}{l}\text { Sim } \\
\text { Näo } \\
\text { O que seu companheiro acha } \\
\text { de amamentar seu filho } \\
\text { Importante, quer que } \\
\text { amamente }\end{array}$ & $\begin{array}{l}80 \\
20\end{array}$ & $\begin{array}{l}80 \% \\
20 \%\end{array}$ \\
\hline Nunca disse nada sobre & 11 & $11 \%$ \\
\hline
\end{tabular}

Fonte: Dados da pesquisa, 2016.

$\mathrm{O}$ alto porcentual de mulheres que realizaram o pré-natal $(100 \%)$ pode ser o reflexo de assistência de saúde na comunidade estudada, mas também fruto do local de onde os dados foram coletados, uma vez que as mães que buscaram a assistência têm uma meIhor visão do autocuidado, e também do atendimento especializado à mãe e ao bebê devido às informações que receberam durante o pré- natal.

Cesar et al. (2012) identificaram, em seu estudo, que a morbimortalidade materno-infantil pode ser reduzida por meio de uma oferta de um pré-natal adequado. A quantidade de consultas está ligada ao melhor resultado gestacional, o que se expressa em bom crescimento intrauterino, maior ganho de peso ao nascer, baixa ocorrência de prematuridade, mortalidade neonatal e de adoecimento e morte entre mães (CESAR et al., 2012).

Em relação aos dados de acompanhamento do pré-natal, foi observado que $84 \%$ das consultas foram realizadas na rede básica de saúde. Um estudo de Silva et al. (2008) mostra que a orientação dada às gestantes, tanto na rede pública quanto na privada, são iguais, porém, ao ser comparado às mães que amamentam exclusivamente até os seis meses do bebê, na rede pública e na rede particular, há uma porcentagem maior nas mães que realizam o pré-natal na rede pública. 
Considerando as informações prestadas ao pré-natal, percebe-se que $70 \%$ das mulheres foram orientadas por um enfermeiro. A consulta de enfermagem proporciona orientações de medidas que visam à abordagem das necessidades das mulheres com as quais os profissionais interagem durante as consultas de pré-natal da unidade básica de saúde. A comunicação entre profissional-cliente é um recurso indispensável para a assistência à saúde, que estabelece confiança e vínculo do usuário ao profissional e, respectivamente, ao serviço de saúde. $O$ trabalho de enfermagem está centrado no cuidado e tem como sujeito o cliente (BARBOSA; GOMES, 2011).

O enfermeiro tem importante papel nos programas de educação em saúde durante o pré-natal, pois é o profissional que se relaciona mais estreitamente com a mulher durante seu ciclo gravídico e puerperal. Ele deve, contudo, preparar a gestante para o aleitamento materno para que no pós-parto a adaptação da puérpera quanto ao aleitamento seja tranquilo e facilitado, evitando dúvidas, possíveis complicações e dificuldades (COUTINHO et al., 2014).

Ainda, Costa (2016) relata que a consulta de enfermagem apresenta-se como instrumento relevante especialmente para a promoção do aleitamento materno, pois esse garante a extensão da cobertura e melhoria da qualidade do pré-natal mediante ações preventivas e promocionais à gestante. $\mathrm{O}$ enfermeiro, além da competência técnica, deve dispor de habilidades de comunicação, escuta e ação dialógica, sendo sensível para compreender a gestante.

No que se refere aos conhecimentos sobre as vantagens da amamentação, $80 \%$ das lactantes relataram conhecê-los. Dias et al. (2016) relatam, em seu estudo, que a grande maioria acredita que há benefícios para a mulher que amamenta seu bebê e as mesmas sempre frisam em seus discursos a proteção contra as doenças tanto para a criança quanto para a mãe. Primo e Leite (2015) afirmam que as mulheres estão cientes que amamentando estão prevenindo doenças, ajudando na dentição do bebê e diminuindo os riscos de desenvolver o câncer de mama.

Em relação ao questionamento do que os companheiros acham sobre amamentar, $84 \%$ das lactantes afirmam que eles acham o ato de amamentar importante. Estes dados corroboram o estudo de Silva et al. (2012), que mostra que quase a totalidade dos pais, $95,4 \%$, apresentou opinião favorável. Uma pesquisa de Ferraz et al. (2016) expõe que o leite materno é percebido pelos pais como um elemento imprescindível à saúde da criança e é extremamente benéfico nos primeiros meses de vida, revelando que os familiares têm a devida informação que o leite materno é uma fonte de promoção à saúde e prevenção de doenças. Segundo Primo e Leite (2015), o apoio familiar é essencial para o início e a continuidade do aleitamento materno, e as principais figuras apoiadoras para este processo são as avós e os companheiros.

Referente às dúvidas sobre amamentar, quando questionadas $99 \%$ das mães afirmaram não apresentar dúvidas, o que nos leva a considerar que, apesar dos dados estarem em controvérsia, acredita-se que tinham dúvidas, porém por fatores pessoais não queriam relatá-los, como, por exemplo, vergonha de falar de seus anseios ou por estarem realizando cuidados e técnicas inadequadas, por não aceitarem a opinião de terceiros ou por não querer estender o seu tempo ouvindo explicações.

Relacionado ao $1 \%$ de dúvida, quando a mãe questionou se poderia fazer o uso de álcool durante a amamentação, Carvalho e Gomes (2017), relatam que o etanol é uma substância depressora do Sistema Nervoso Central. Apesar de uma quantidade significativa ser excretada no leite, ela não é considerada perigosa ao lactente, em doses e períodos limitados. A ingestão de $0,3 \mathrm{~g} / \mathrm{kg}$, conteúdo presente em uma lata de cerveja (350 ml), no entanto, pode reduzir em até $23 \%$ a ingestão do leite pela criança. Além disso, pode causar o odor e a mudança do sabor do leite materno, levando à recusa pela criança.

\section{CONSIDERACÕES FINAIS}

A realização desta pesquisa possibilitou identificar o perfil das lactantes e o conhecimento que as mesmas têm sobre a importância do aleitamento materno exclusivo para a saúde do bebê. As mães atendidas na rede básica de saúde se resumem em lactantes jovens, a grande maioria vivendo com seu companheiro, possuem o Ensino Médio completo e não apresentam vínculo empregatício. Diante dos antecedentes obstétricos, observamos que a maioria são primíparas e relataram não possuir experiência em amamentar.

Infere-se que as lactantes entrevistadas podem não ter apresentado dúvidas por vergonha de relatar os fatos ou por medo de estarem realizando cuidados e técnicas erradas e por não aceitarem opinião alheia. Essa foi uma das limitações do estudo, pois a incidência na resposta, quando dizem não apresentarem dúvidas, relacionando ao fato de que essas mães são primigestas e disseram ter pouca experiência em amamentar, nos leva a acreditar que elas não responderam de forma coerente e fidedigna ao estudo. 
Sugere-se que sejam realizados novos estudos na área da amamentação, pesquisando-se a relação entre as dificuldades que lactantes mais jovens e adultas primíparas apresentam, quais fatores interferem criticamente na amamentação exclusiva e qual intervenção pode ser realizada para amenizar ou erradicar a interrupção do aleitamento materno até os seis meses.

Conclui-se que as mães possuem conhecimento sobre amamentação e, mesmo a maioria sendo jovens e primigestas e apresentando algumas dificuldades, elas sempre estão dispostas a dar o seu meIhor para o filho. Assim, mostra-se a importância do acompanhamento do enfermeiro durante o pré-natal, preparando-as para o momento da amamentação e orientando-as quanto às dúvidas que surjam desde o início da gestação até os primeiros meses do bebê, para que o puerpério e o aleitamento materno sejam um momento agradável e sem maiores dificuldades.

\section{REFERÊNCIAS}

BARBOSA, T. L. A.; GOMES, L. M. X.; DIAS, O. V. O pré-natal realizado pelo enfermeiro: a satisfação das gestantes. Revista Cogitare Enfermagem, 16(1), p. 29-35, jan./mar. 2011.

BRASIL. Ministério da Saúde. Secretaria de Atenção à Saúde. Departamento de Atenção Básica. Saúde da Criança: nutrição infantil. Aleitamento materno e alimentação complementar. Brasília: Ministério da Saúde, 2009.

CARVALHO, M. R. de; GOMES, F. C. Amamentação - base cientifica. 4. ed. Rio de Janeiro: Ed. Guanabara, 2017.

CESAR, J. Á. et al. Assistência pré-natal nos serviços públicos e privados de saúde: estudo transversal de base populacional em Rio Grande, Rio Grande do Sul. Brasil. Cad. Saúde Públ., 28(11), p. 2.106-2.114, nov. 2012.

COIMBRA, L. C.; RIETH, N. F. de A. Caracterização do aleitamento materno em São Luís, Maranhão. Rev. Pesq. Saúde, 17(1), p. 7-12, jan./abr. 2016.

COUTINHO, E. de C. et al. Gravidez e parto: o que muda no estilo de vida das mulheres que se tornam mães? Rev. Esc. Enferm. USP, v. 48, (Esp. 2), p. 17-24, 2014.

COSTA, D. A. da. Atribuições do enfermeiro durante a consulta pré-natal relacionadas ao aleitamento materno: relato de experiência. 2016. 28f. Trabalho de Conclusão de Curso (Graduação em Enfermagem) - Universidade Estadual da Paraíba, Campina Grande, 2016.

DIAS, E. G. et al. Vantagens da amamentação e alterações no estilo de vida da lactante. Revista Contexto \& Saúde, v. 16, n. 31, 2016.

. Prevalência do aleitamento materno exclusivo até o sexto mês no município de Mamonas - MG em 2013. Revista Contexto \& Saúde, ljuí: Ed. Unijuí, v. 15 n. 29, 2015.

FERRAZ, L. et al. Opinião de mulheres sobre a participação do pai no aleitamento materno. Arq. Cienc. Saúde Unipar, Umuarama, v. 20, n. 2, p, 95-99, maio/ago. 2016.

MELO, Sheila Caroline Hnediuk de; SCHERMANN, Lígia Braun. Prevalência e fatores associados à dificuldade no cuidado do bebê em mães adolescentes de 14 a 16 anos de Porto Alegre/RS. Aletheia, n. 38-39, p. 67-80, maio/dez. 2012.
MOLINA, F. R.; GIL, N. L. M.; VICTORIANO, S. V. Z. Prevalência do aleitamento materno exclusivo no município de Marialva-Paraná. Revista Uningá, Maringá-PR, n. 38, p. 71-83, out./dez. 2013.

NOGUEIRA, Cibele Mary Ramos. Conhecimento sobre aleitamento materno de parturientes e prática de aleitamento cruzado na Unidade Hospitalar e Maternidade Venâncio Raimundo de Souza - Horizonte - Ceará. Rio de Janeiro: [s.n.], 2009. 58 p.

OLIVEIRA, C. N. T.; OLIVEIRA, M. V. Prevalência de aleitamento materno exclusivo e fatores associados ao desmame precoce no município de Vitória da Conquista-BA. C\&D-Revista Eletrônica da Fainor, Vitória da Conquista, v. 5, n. 1, p. 160-174, jan./dez. 2012.

ORGANIZAÇÃO MUNDIAL DA SAÚDE. OMS. Saúde da criança: aleitamento materno e alimentação complementar. Disponível em: <http://bvsms.saude.gov.br/bvs/publicacoes/saude_crianca_aleitamento_materno_cab23.pdf $>$. Acesso em: maio 2017.

PRIMO, C. C.; LEITE, F. M. C. Redes sociais que apoiam a muIher durante a amamentação. Vitória, ES: Universidade Federal do Espírito Santo, 2015.

REIS, J. R. G.; GONÇALVES, L. C. S. Fatores relacionados ao desmame precoce. Revista Perquirere, 13 (2), p. 218-228, dez. 2016.

ROCCl, E.; FERNANDES, R. A. Q. Dificuldades no aleitamento materno e influência no desmame precoce. Rev. Bras. Enferm., Brasília, v. 67, n. 1, jan. 2014.

SANTOS, E. H. O desafio da amamentação para puérperas adolescentes. Criciúma/SC, dez. 2012 (Pesquisa apresentada à disciplina de Elaboração Acadêmica de Artigo, do Curso de Especialização em Enfermagem Pediatria e Neonatalologia do Instituto de Pós-Graduação de Criciúma - IPG/ Esucri).

SILVA, I. M. D et al. Técnica da amamentação: preparo das nutrizes atendidas em um hospital escola, Recife-PE. Rev Rene, Fortaleza, (n. esp.), p. 1.021-1.027, 2012.

SILVA, P. P. A percepção das mães sobre o apoio paterno: influência na duração do aleitamento materno. Rev. Paul. Pediatr., 30(3), p. 306-313, 2013.

SILVA, M. O. C et al. Aleitamento Materno Exclusivo (AME), pré-natal particular e pré-natal público: estudo de casos cadastrados em um Programa de Saúde da Família do sul fluminense. Caderno Unifoa, edição especial prefeitura de Volta Redonda, out. 2008.

SOUZA, J. P. B.; MENDES, L. L.; BINOTI, M. L. Perfil do aleitamento materno e da alimentação complementar em crianças menores de dois anos atendidas em um centro de referência da cidade de Juiz de Fora - MG. Rev. APS., 19(1), p. 67-76, jan./mar. 2016.

TETER, M. S. H.; OSELAM, G. B.; NEVES, E. B. Amamentação e desmame precoce em lactantes de Curitiba. Revista Espaço Para a Saúde, Londrina, v. 16, n. 4, p. 55-63, out./ dez. 2015.

VISINTIN, A. B. et al. Avaliação do conhecimento de puérperas acerca da amamentação. Enferm. Foco, n. 6, 2015. 